\title{
Ejection of the inner accretion disk in GRS 1915+105: The magnetic rubber-band effect
}

\author{
A. Nandi ${ }^{1}$, S. K. Chakrabarti ${ }^{1,2}$, S. V. Vadawale ${ }^{3}$, and A. R. Rao ${ }^{3}$ \\ 1 S.N. Bose National Center for Basic Sciences, Salt Lake, 700 098, Kolkata, India \\ 2 Centre for Space Physics, 114/v/1A Raja S.C. Mullick Rd., 700047, Kolkata, India \\ 3 Tata Institute of Fundamental Research, Homi Bhabha Road, 400 005, Mumbai (Bombay), India
}

Received 19 April 2001 / Accepted 4 October 2001

\begin{abstract}
We examine theoretically the behaviour of the inner accretion disk in GRS 1915+105 when soft X-ray dips are present in the X-ray light curve. We assume the presence of a radial shock in the accretion disk, as in some of the Two Component Advective Flow (TCAF) solutions. We discuss the behaviour of the flux tubes inside a TCAF (which we name Magnetized TCAF or MTCAF model for brevity) and compare various competing forces on the flux tubes. In this MTCAF model, we find that the magnetic tension is the strongest force in a hot plasma of temperature $\gtrsim 10^{10} \mathrm{~K}$ and as a result, magnetic flux tubes entering in this region collapse catastrophically, thereby occasionally evacuating the inner disk. We postulate that this magnetic "rubber-band" effect induced evacuated disk matter produces the blobby components of outflows and IR/radio jets. We derive the size of the post-shock region by equating the time scale of the Quasi-Periodic Oscillations to the infall time of accreting matter in the post-shock region and found the shock location to be $\sim 45-66 r_{\mathrm{g}}$. We calculate the transition radius $r_{\mathrm{tr}}$, where the Keplerian disk deviates into a sub-Keplerian flow, to be $\sim 320 r_{\mathrm{g}}$. Based on the derived X-ray spectral parameters, we calculate the mass of this region to be $\sim 10^{18} \mathrm{~g}$. We conclude that during the X-ray dips the matter in the post-shock region, which manifests itself as the thermal-Compton component in the X-ray spectrum, is ejected, along with some sub-Keplerian matter in the pre-shock region.
\end{abstract}

Key words. accretion, accretion disks - black hole physics - stars: winds, outflows - stars: individual: GRS1915+105 - X-rays: stars

\section{Introduction}

GRS 1915+105 has proven to be an ideal source to study in detail many of the physical concepts regarding accretion onto black holes. Ever since its discovery (Castro-Tirado et al. 1992), it has been continuously bright in X-rays and it shows a variety of X-ray variability characteristics (Morgan et al. 1997; Muno et al. 1999; Yadav et al. 1999; Belloni et al. 2000). It has been monitored extensively in the radio band (Mirabel \& Rodriguez 1994; Pooley \& Fender 1997; Fender et al. 1999) and several episodes of high radio emissions, huge flares associated with superluminal motions, radio oscillations etc. are observed in this source. Several attempts have been made to associate the radio emission, presumably coming from jets, to the X-ray emission from the accretion disks (Fender et al. 1999; Naik et al. 2001; Naik \& Rao 2001). The Spectral signature of winds from the Comptonising region has also been identified (Chakrabarti et al. 2001).

Send offprint requests to: A. Nandi, e-mail: anuj@boson.bose.res.in
Chakrabarti \& Manickam (2000, hereafter CM00) have applied the Two Component Advective Flow (TCAF) model of Chakrabarti \& Titarchuk (1995) to explain various X-ray variability characteristics of GRS $1915+105$. Recently there has been overwhelming evidence that the TCAF model is valid for many black hole candidates (Smith et al. 2001a; Smith et al. 2001b). CM00 invoked outflows from the inner accretion disk to explain a correlation between the QPO frequency and the duration of the burst-off states during a regular oscillations seen in the source. These outflows, however, are confined to the sonic sphere and fall back on the accretion disk after being cooled down by an inverse Compton effect. It was pointed out by Naik \& Rao (2001) that the source does not show appreciable radio emission during such oscillations. A detailed inflow/outflow model has not been presented for this source to explain the radio emission, particularly to explain the superluminally moving radio blobs.

Recently Naik et al. (2001) have detected a series of soft X-ray dips during the declining phase of a huge radio flare and have postulated that such soft dips are responsible for the jet emission. Vadawale et al. (2001) made 
a detailed study of X-ray dips observed during the radio flare using the Rossi X-ray Timing Experiment (RXTE) data and have presented evidence for the disappearance of the inner accretion disk during the dips. Since the disappearance of the inner disk is seen to be correlated with intense radio activity, the role of the magnetic field must be studied in order to understand the system completely. Rodriguez \& Mirabel (1999) estimated the field in radio blobs to be around tens of $\mathrm{mG}$ at 500-1000 AU (in 1994 observation). Fender et al. (1997) requires the field to be around $8 \mathrm{G}$ at around $1 \mathrm{AU}$ (in their 1996 observations). From the similarity of $\sim 30 \mathrm{~min}$ oscillations in IR and Radio, they concluded that the radio blobs are adiabatically expanding and are independently ejected from the disk every 30-40 min. If the trapped field inside a radio blob is of roughly $1 / r$ (for toroidal field) then its interpolated value close to a black hole is around $10^{7} \mathrm{G}$ at around $10 r_{\mathrm{g}}$ which is comparable to an equipartition value. Thus, one needs to correlate fields ejected from the disk with those observed inside the radio blobs. In this paper, we examine the mass ejection based on the TCAF model in presence of a magnetic field (we call this as Magnetized TCAF or MTCAF model) amplified due to strong shear at the transition radius of the Keplerian and a sub-Keplerian flow. In the next section, we discuss forces which govern the motions of the flux tubes and write equations of toroidal flux tubes inside an accretion disk with a constant angular momentum. We show that close to the black hole, where the flow could be very hot $\left(\gtrsim 10^{10} \mathrm{~K}\right)$ the flux tubes move at least with the Alfvén speed and may catastrophically collapse like a stretched rubber band. We conjecture that such rapid collapse would assist evacuation of matter from the disk and cause X-ray "dips" seen in the light curves. In Sect. 3 we estimate the mass of the ejecta which agrees with observations. Finally, in Sect. 4 we draw our conclusions.

\section{The magnetized TCAF model for GRS $1915+105$}

Based on the global solutions of the most general advective accretion disk solution (Chakrabarti 1990, 1996a), Chakrabarti \& Titarchuk (1995) presented a TCAF model of accretion onto black holes. According to this model, matter with high viscosity and angular momentum settles into the equatorial plane, while matter with lower viscosity and angular momentum surrounds the Keplerian disk. This sub-Keplerian component is likely to form a standing or an oscillating shock (Molteni et al. 1996; Ryu et al. 1997) front near the centrifugal barrier (few tens of Schwarzschild radii) depending on whether the RankineHugoniot condition is satisfied or the cooling time in the post-shock region is comparable with the infall time. Soft photons from the Keplerian disk in the pre-shock flow are intercepted by the puffed up sub-Keplerian post-shock flow and are reprocessed due to inverse Comptonization. If the post-shock matter remains hot, the black hole exhibits a harder spectrum, while if the post-shock region is

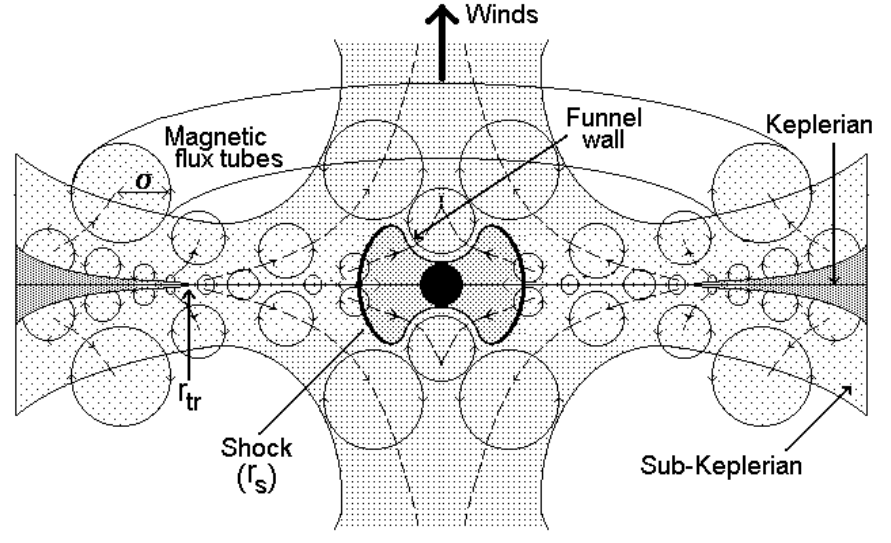

Fig. 1. A cartoon diagram of the accretion disk near a black hole which includes a shock $\left(r_{\mathrm{s}}\right)$, a sub-Keplerian and a Keplerian disk with a boundary at $r_{\mathrm{tr}}$. Stochastic magnetic fields are sheared and amplified as they leave a Keplerian disk. In a hot, sub-Keplerian flow, these toroidal flux tubes catastrophically collapse squirting matter along the axis, and thereby evacuating the disk and producing outflows. Dashed curves show typical trajectories (CD94).

cooled down by the soft photons, the black hole exhibits a soft spectrum. There could be a sub-Keplerian region just before the shock as well since with low viscosity and accretion rates a Keplerian disk recedes from a black hole and it is not necessary that the shocks form right at the region where a transition from Keplerian to sub-Keplerian flow takes place. Figure 1 schematically shows this behaviour of the two components.

The centrifugal pressure supported boundary layer (or, CENBOL for short) formed in a transonic, advective flow (Chakrabarti et al. 1996), has most of the features of a thick accretion disk although in advective disks, advection is included self-consistently and in thick disks advection is totally ignored. This is because at the CENBOL surface, matter undergoes a supersonic to sub-sonic transition and it moves very slowly in the radial direction. In some phases of accretion, matter can bring in a large stochastic magnetic field. The field is sheared due to strong azimuthal velocity and the toroidal field becomes very strong. These field lines will have very little matter within it and would likely be buoyant and emerge from various parts of the accretion disk. Figure 1 schematically shows this behaviour. Toroidal magnetic flux tubes released from the Keplerian disk are sheared, amplified and are advected in the subKeplerian flow. Due to the centrifugal barrier matter stays away from the axis. Thus a so-called funnel wall is created exactly as in a thick accretion disk (Paczyński \& Wiita 1980). Chakrabarti \& D'Silva (1994, hereafter CD94) computed the nature of their trajectories inside a thick accretion disk. They showed that in the event a strong flux tube enters a hot region with ion temperature $T_{\mathrm{i}} \gtrsim 10^{10} \mathrm{~K}$, the magnetic tension becomes the strongest force and the flux tube catastrophically collapses. Because of strong similarity of the thick accretion disk and an advective flow, especially inside the CENBOL, we believe that a similar 
mechanism could be working and flux tube collapse would take place.

We shall consider the motion of the flux tubes on the equatorial plane of an accretion flow around a Schwarzschild black hole described by Paczyński-Wiita (1980) pseudo-Newtonian acceleration $g=-1 /\left[2(x-1)^{2}\right]$. We use the geometric units. Masses are measured in units of the mass of the central black hole, $M_{\mathrm{BH}}$; distances from the axis $(x)$ will be measured in units of the Schwarzschild radius $r_{\mathrm{g}}=2 G M_{\mathrm{BH}} / c^{2}$; and the time scales are measured in units of $r_{\mathrm{g}} / c$. Inside the disk, we choose the polytropic equation of state, $P=K \rho^{\gamma}$, where $K$ and $\gamma$ are constants. The magnetic flux tubes brought in by advection are assumed to be sheared and axisymmetric toroidal flux tubes of random shape and size could be produced inside the flow. We however assume that the flux tubes are thin, i.e., the flux tube cross-sectional radius $\sigma$ is smaller than the local pressure scale height of the disk. Close to a black hole, angular momentum of the flow remains constant (Chakrabarti 1996a) even in presence of moderate viscosity. Thus, we choose specific angular momentum $\lambda$ in the sub-Keplerian region to be constant. The equations of motion for thin flux tubes have been given in CD94 and we do not repeat here. For the sake of completeness, we write down the radial equation only valid for the equatorial plane $(\theta=\pi / 2)$,

$$
\begin{aligned}
\ddot{x}+ & \frac{X}{(1+X)}\left[-x \dot{\phi}^{2}-2 x \omega \dot{\phi}\right]= \\
& \frac{X}{(1+X)}\left\{\frac{M_{\mathrm{b}}}{X}\left[g-x \omega^{2}\right]-\frac{1}{m_{\mathrm{i}}} \frac{\psi^{2}}{2 \pi \sigma^{2}}-\frac{D_{x}}{\pi \sigma^{2} \rho_{\mathrm{e}}}\right\},
\end{aligned}
$$

where $X=m_{\mathrm{i}} / m_{\mathrm{e}}, m_{\mathrm{i}}=2 \pi^{2} \sigma^{2} x \rho_{\mathrm{i}}$ and $m_{\mathrm{e}}=2 \pi^{2} \sigma^{2} x \rho_{\mathrm{e}}$ are the masses of the fluid inside and the fluid displaced by the flux tube respectively, $\rho_{\mathrm{i}}$ and $\rho_{\mathrm{e}}$ being the corresponding densities. Subscripts e and i indicate whether the relevant quantity is of the ambient (external) medium or within the flux tube (internal medium). $\psi=\pi \sigma^{2} B, B$ being magnetic field of the tube and $\dot{\phi}$ is the intrinsic angular velocity of the flux tube inside the disk. The drag term is assumed to be similar to the drag experienced by a cylinder moving perpendicular to its axis in a fluid, and the drag per unit length in radial direction is given by,

$D_{x}=-\frac{1}{2} C_{\mathrm{D}} \rho_{\mathrm{e}} \sigma(\dot{x}-u)^{2}$

where $C_{\mathrm{D}} \sim 0.4$ (Goldstein 1938). The pre-shock, subKeplerian flow is assumed to have a radial velocity,

$$
u \sim \beta / \sqrt{(x-1)}
$$

and the post-shock sub-Keplerian flow,

$$
u \sim 1 / R \sqrt{(x-1)} .
$$

Here, $\beta$ is a factor by which sub-Keplerian matter slows down compared to a freely falling flow. $\beta \sim 1$ for very low angular momentum cool flow. $R$ is the compression ratio of the shock by which matter is assumed to be slowed down inside the CENBOL.
From Eq. (1), we note that there are four forces in operation: (i) The second term inside the bracket of the left hand side is the Coriolis force $F_{\mathrm{c}}=2 v_{\phi} \omega$, where $\omega=\lambda / x^{2}$ is the angular velocity of the flow. The Coriolis acceleration is given by (CD94),

$a_{\mathrm{C}}=\frac{\rho_{\mathrm{i}}}{\rho_{\mathrm{i}}+\rho_{\mathrm{e}}} \frac{2 \lambda^{2}}{x}\left[\frac{1}{x_{0}^{2}}-\frac{1}{x^{2}}\right]$.

A flux tube brought from $x_{0}$ to $x$ inside a flow of constant angular momentum would feel no Coriolis force if the motion is along the direction of constant angular velocity since $x_{0} \sim x$ for rotating bodies. A magnetic flux tube with buoyancy factor $M_{\mathrm{b}}\left(=\frac{\rho_{\mathrm{e}}-\rho_{\mathrm{i}}}{\rho_{\mathrm{e}}}\right)=1-X$, will feel the (ii) Magnetic Buoyancy force (first term on the right hand side inside the curly bracket). The corresponding acceleration is (CD94):

$a_{\mathrm{MB}}=\frac{M_{\mathrm{b}}}{1+X}\left[\frac{1}{2(x-1)^{2}}-\frac{\lambda^{2}}{x^{3}}\right]$.

The tube will emerge out of the disk if $a_{\mathrm{MB}}$ dominates over $a_{\mathrm{C}}$. The middle term on the right hand side of Eq. (1) is the (iii) force due to Magnetic Tension. The corresponding acceleration is $a_{\mathrm{T}}(\mathrm{CD} 94)$ :

$a_{\mathrm{T}}=-\frac{1}{m_{\mathrm{i}}+m_{\mathrm{e}}} \frac{\Psi^{2}}{2 \pi \sigma^{2}}=-\frac{B^{2}}{4 \pi x\left(\rho_{\mathrm{i}}+\rho_{\mathrm{e}}\right)}$.

The final and very important force is represented by the final term of Eq. (1). It is called the (iv) Drag Force exerted due to the motion of a rigid tube inside a flow. The corresponding acceleration is (CD94):

$a_{\mathrm{D}}=-\frac{C_{\mathrm{D}} \rho_{\mathrm{i}} \sigma(\dot{x}-u)^{2}}{2 \pi \sigma^{2}\left(\rho_{\mathrm{i}}+\rho_{\mathrm{e}}\right)} \sim-\frac{C_{\mathrm{D}} \rho_{\mathrm{i}} u^{2}}{2 \pi \sigma\left(\rho_{\mathrm{i}}+\rho_{\mathrm{e}}\right)}$.

Here we used $\dot{x} \sim 0$ for computing the maximum value of the drag force.

Equating the acceleration due to buoyancy with that due to magnetic tension (Eq. (6)) on the equatorial plane, we get the critical temperature of the external gas as (CD94),

$T_{\mathrm{p}, 0}=\frac{m_{\mathrm{p}} c^{2}\left[\lambda_{\mathrm{K}}^{2}\left(x_{0}\right)-\lambda^{2}\right]}{4 k x_{0}^{2}}$,

above which the tension dominates over buoyancy and the flux tubes rush towards the funnel wall catastrophically. Here we used the mean electron number per ion to be $\mu=1 / 2$ and $k$ is the Boltzmann constant. Subscript 0 specifically indicates that the flux tube will behave like an over stretched rubber-band only after it crosses $x=x_{0}$, where $T>T_{\mathrm{p}, 0}$. Note that since we are dealing with a sub-Keplerian disk, $T_{\mathrm{p}, 0}>0$ in the entire region of interest. For typical values $x_{0}=100, \lambda_{0}=1.8$, one obtains $T_{\mathrm{p}, 0} \sim 6 \times 10^{9} \mathrm{~K}$. For an adiabatic disk, this assumption remains valid even when the flux tube collapses very rapidly since its internal temperature will increase adiabatically in the same way as in the external disk. For an efficiently cooled two-temperature flow, the above proton 
temperature would correspond to an electron temperature of $T_{\mathrm{e}} \sim \sqrt{m_{\mathrm{e}} / m_{\mathrm{p}}} T_{\mathrm{p}}$. This is around $14 \mathrm{keV}$ which is very reasonable for the temperature of the sub-Keplerian region. When the flux tubes fall radially, neither Coriolis force nor the drag term could be neglected. In fact, accelerated flux tubes would have high $\dot{x}$ in the drag term as they move faster than the bulk radial motion. Meanwhile, assuming that internal mass of a flux tube is roughly constant, the cross-section $\sigma \sim 1 / \sqrt{x}$ increases as the flux tube approaches the black hole. So, this will also increase the drag term. As a result, we expect that the flux tube would slow down somewhere close to the black hole and buoyancy would eject the flux tube out of the disk perpendicularly as shown in Fig. 1. Typical trajectories of the flux tubes, based on numerical integrations in CD94, are shown in Fig. 1.

The buoyancy timescale $t_{\mathrm{b}}$ is estimated from Eq. (5): $a_{\mathrm{MB}} \sim h / t_{\mathrm{b}}^{2}=\frac{M_{\mathrm{b}}}{(1+X) x^{3}}\left(\lambda_{\mathrm{K}}^{2}-\lambda^{2}\right)$, where, $h \sim a_{\mathrm{s}} x^{3 / 2}$ is the half thickness of the disk in vertical equilibrium at $x$ and $a_{\mathrm{s}} \sim x^{-1 / 2}$ is the speed of sound. Note that $t_{\mathrm{b}}$ is very large in a Keplerian disk $\left(\lambda_{\mathrm{K}} \sim \lambda\right)$. For a sub-Keplerian flow, $\lambda / \lambda_{\mathrm{K}} \sim 0.4$ with $\lambda=1.8$ at $x=30, X \sim 0.1, M_{\mathrm{b}} \sim 0.9$, $t_{\mathrm{b}} \sim 240$.

Collapse time $t_{\mathrm{f}}$ of a flux tube is estimated in the following way: when tension is the most dominant force, the radial equation of the flux tube (Eq. (1)) is simplified to:

$\ddot{x}+a_{\mathrm{T}}=0$,

Putting $\ddot{x} \sim x / t_{\mathrm{f}}^{2}$, we see that the velocity of collapse of the flux tube $v_{\mathrm{f}}$ is,

$v_{\mathrm{f}} \sim\left(\frac{B^{2}}{4 \pi \rho_{\mathrm{e}}}\right)^{1 / 2} \sim v_{\mathrm{a}}$

where $v_{\mathrm{a}}$ is the Alfven velocity. Thus, flux tube collapses in Alfv́en speed. Since $B \propto x^{-2}$, magnetic pressure is $\propto x^{-4}$, while the gas pressure $p_{\mathrm{e}} \propto x^{-5 / 2}$, the ratio of magnetic to gas pressure $f \propto x^{-3 / 2}$, increasing with the decrease of $x$. As the flux tube leaves the Keplerian disk, large shear at the transition region and in the sub-Keplerian flow quickly intensifies the flux tube to $M_{\mathrm{b}} \sim 1$ during infall. On the other hand, if $f \sim 1$ at $x \sim 300$ where the flow deviates from a Keplerian disk, $f \sim 30$ at $x=30$, inside the CENBOL. The Alfv́en speed $v_{\mathrm{a}} \sim\left(\frac{B^{2}}{4 \pi \rho_{\mathrm{e}}}\right)^{1 / 2} \sim$ $(f / \gamma)^{1 / 2} a_{\mathrm{s}} \sim 5 a_{\mathrm{s}}$ where we used $\gamma=4 / 3$. Since, inside a CENBOL, the velocity of matter $v_{\mathrm{m}}$ is highly sub-sonic, it is therefore high sub-Alfv́enic: $v_{\mathrm{m}} \ll v_{\mathrm{a}}$. Thus, $v_{\mathrm{f}} / v_{\mathrm{m}} \gg 1$ and the flux tube collapses catastrophically. This justifies the conjecture made earlier that the rubber-band effect could evacuate the disk (Chakrabarti 1994, 1996b, 2000). For $a_{\mathrm{s}} \sim x^{-1 / 2}, t_{\mathrm{f}} \sim x^{3 / 2} / 5 \sim 30$ at $x=30$. Thus inside the CENBOL $t_{\mathrm{b}} \gg t_{\mathrm{f}}$.

So far, we have ignored many non-axisymmetric effects such as Parker instability (1979 and references therein) and shearing instability (e.g., Balbus \& Hawley 1991). Foglizzo \& Tagger (1995) treated this problem comprehensively in the context of a standard disk embedded in a large scale field. They found that (a) if the wave-length is larger than the disk thickness then the flux tube is very unstable and buoyantly comes out of the disk and (b) instability is strongest if the field is weaker. According to Parker (1979), submerged field tubes may break up into filaments in timescales of around $3 \Lambda / v_{\mathrm{a}}$, where $\Lambda$ is the scale height and the field is able to escape from the gas in timescales $t_{\mathrm{P}} \sim \Lambda / v_{\mathrm{a}}$. If $\Lambda \sim h \sim x$, which is especially true in CENBOL region, the time scale of the escape of the field may be comparable to the $t_{\mathrm{f}}$ as obtained above. As a result, the flux not only collapses catastrophically, but also escapes upwards following a curved trajectory as depicted in Fig. 1. In presence of a differential rotation, Balbus \& Hawley (1991) suggested that even a small initially vertical field would be amplified to create all the components in the dynamical timescale $t_{\mathrm{d}} \sim 1 / \omega=x^{2} / \lambda \sim 50$ (at $x=30, \lambda=1.8$ ). Numerical simulation (Hawley et al. 1995) has verified this instability neglecting the tension effects. Even though the time scales of the collapse, the Parker instability and the shear instability are of the same order, we believe that the rubber-band effect would still be important for the destruction of the inner disk.

\section{Estimation of mass of the post-shock region and the sub-Keplerian region}

Vadawale et al. (2001) have shown that during the soft $\mathrm{X}$-ray dips a thermal-Compton component in the X-ray spectrum gets suppressed. Several works in the literature talk about the disk-evacuation (e.g., Belloni et al. 1997; Feroci et al. 1999) in this context. We like to understand this using Two Component Advective Flow (TCAF) models of Chakrabarti \& Titarchuk (1995) in presence of a Magnetic field (i.e., MTCAF model) and its time variability properties described in Molteni et al. (1996); Ryu et al. (1997) and Chakrabarti \& Manickam (2000). The observation of possible disk-evacuation is clearly in line with the TCAF model and the shock oscillation model of the quasi-periodic oscillations (CM00; see also, Rao et al. 2000) which showed that the Comptonising post-shock region participates in oscillation. The sub-Keplerian region in the pre-shock flow does not emit much radiation and it is possible that some of this region may also be disrupted during the rapid collapse of the flux tube. Once we accept the destruction of the sub-Keplerian region by the magnetic rubber-band effect, we can compute the mass of this region in the following way:

The shock location is computed by equating the infall time from the shock with the time scale of QPO. This time scale $t_{\mathrm{ff}}$ can be written in the form (CM00):

$t_{\mathrm{ff}}^{-1}=\frac{1}{R} \frac{1}{r_{\mathrm{s}}^{\alpha}} \frac{c v_{0}}{r_{\mathrm{g}}}$

where, $R$ is the compression ratio (see also, Eq. (3b)), $r_{\mathrm{s}}$ is the shock location, $v_{0}$ is a dimensionless constant. Here, $\alpha=3 / 2$ for free-fall motion and $\alpha=1$ for a flow of constant velocity $c v_{0} / R$ in the post-shock region. Using this assumption, the shock location in the pre-dip and the post-dip flow (for parameters in these flow see, 
Vadawale et al. 2001) are $\sim 45 r_{\mathrm{g}}$ and $\sim 66 r_{\mathrm{g}}$ respectively. CM00 proposes that a better fit in the correlation between the duration of the QPO and the frequency of QPO requires more or less constant velocity in the post-shock region with a rough velocity of $0.066 c / R$. This produces the shock in the pre-dip and the post-dip flow at $79 r_{\mathrm{g}}$ and $140 r_{\mathrm{g}}$ respectively. Using the parameters of Vadawale et al. (2001), and the location of the shock as given above, the electron number density in the Comptonising region is found to be $n_{\mathrm{e}} \sim 10^{17} \mathrm{~cm}^{-3}$. The corresponding mass $\left(\frac{4}{3} \pi r_{\mathrm{s}}^{3} m_{\mathrm{p}} n_{\mathrm{e}} r_{\mathrm{g}}^{3}\right)$ of the region is $M_{\mathrm{CENBOL}} \sim 2-3 \times 10^{18} \mathrm{~g}$ depending on the model of the inflow. How much of this matter is squirted out of the disk along the axis? Again, in the absence of the size distribution of the flux tubes, the answer is difficult. However, because of centrifugal force, matter is unlikely to enter within the "funnel wall" (CD94) even after compression, sudden collapse of large flux tubes $\left[\sigma \sim h\left(r_{\mathrm{s}}\right)\right]$ would be expected to displace the whole CENBOL region parallel to the funnel wall. The outflow rate would be $\dot{M}_{\text {out }} \sim M_{\text {CENBOL }} / t_{\text {f }}$ which may be very large compared to the inflow rate for a short duration of $t_{\mathrm{f}}$. If flux tubes are smaller in size, since the collapse velocity is much larger than the speed of sound, matter will still be displaced but would be refilled in free-fall time, unless there are many near-simultaneous flux collapse events.

If the magnetized sub-Keplerian disk is removed by imploding flux tubes as described in the earlier section, one requires to know the location of the inner edge of the Keplerian disk to estimate the complete mass involved. From the model fit (Vadawale et al. 2001), the Keplerian disk temperature turns out to be $T_{\mathrm{K}} \sim 1.5 \mathrm{keV}$. With a hardening factor of around 1.7 (Shimura \& Takahara 1995), the mass of the black hole as $10 M_{\odot}$ and ShakuraSunyaev viscosity parameter $\alpha_{\mathrm{SS}}=0.01$, the above temperature corresponds to a transition radius at around $r_{\text {tr }} \sim 320 r_{\mathrm{g}}$ (Shakura \& Sunyaev 1973). Assuming density falling off as $\rho \sim \rho_{0}\left(\frac{x_{0}}{x}\right)^{3 / 2}$, the mass of the sub-Keplerian flow of size $x_{\operatorname{tr}}\left(\int 4 \pi x h(x) \rho \mathrm{d} x\right.$ with $\left.h(x) \sim x\right)$ is around $10^{20} \mathrm{~g}$. These computations assume no pair production, i.e., there is exactly one electron for each proton in the Comptonising region.

Once the evacuation is complete, the disk is filled in quickly by sub-Keplerian matter in timescale of:

$$
\begin{aligned}
t_{\mathrm{visc}} & \sim \frac{1}{\alpha_{\mathrm{SS}}}\left(\frac{h(x)}{x}\right)^{-2} \frac{x}{v_{\mathrm{Kep}}} \\
& =192\left(\frac{0.01}{\alpha_{\mathrm{SS}}}\right)\left(\frac{0.03}{a_{\mathrm{S}}}\right)^{2}\left(\frac{x_{\mathrm{tr}}}{300}\right)^{1 / 2} \frac{M_{\mathrm{BH}}}{10 M_{\odot}} \mathrm{s} .
\end{aligned}
$$

Here, $h(x) \sim a_{\mathrm{S}} x^{3 / 2}$ ( $a_{\mathrm{s}}$ is the speed of sound) is the local vertical height of the disk. The time scales seem to be reasonable, since the dips have been seen to be filled up in a matter of 150 to $200 \mathrm{~s}$. It is to be noted that Dhawan et al. (2000) using disk-instability model of Belloni et al. (1997) obtained the missing inner disk region to be only $180 \mathrm{~km}$. This distance is about $6 r_{\mathrm{g}}$ and with $10^{18} \mathrm{~g} \mathrm{~m} \mathrm{~s}^{-1}$ accretion rate which they employ, the mass of this region cannot be enough to create the $10^{23} \mathrm{~g}$ m blob (or, even $10^{18} \mathrm{~g}$ miniblobs) observed by Mirabel \& Rodriguéz (1999). This is particularly because the region $1-3 r_{\mathrm{g}}$ is definitely supersonic and sub-Keplerian and therefore is of very little mass. We believe that the missing region should be much larger, possibly order of a $100 r_{\mathrm{g}}$ or so.

\section{Conclusion}

In this paper, we have given a physical basis for a sudden mass ejection in GRS 1915+105. We showed that if matter brings in a particularly strong magnetic field, this would be sheared and amplified to a value much above the equipartition value before it can be expelled by buoyancy. Magnetic tension collapses these toroidal flux tubes at a highly supersonic speed, much faster than the flow velocity. This has the effect of displacing matter from the disk in transverse direction (much like a fast boat causing spillage on a shore) and depositing it to outflowing winds. From the observed fits of Vadawale et al. (2001) we estimated the electron number density and the mass of the post-shock region and the sub-Keplerian flow to be around $10^{18} \mathrm{~g}$ and $10^{20} \mathrm{~g}$ respectively. Our estimate of the post-shock mass is a factor of ten less than the mass estimate $\left(10^{19} \mathrm{~g}\right)$ of "baby-jets" (Mirabel et al. 1998) associated with IR and radio flares and could therefore be due to ejection of some sub-Keplerian matter as well. These "baby-jets" are found to be associated with class $\beta$ light curves which have soft X-ray dips. These dips are also seen in class $\theta$ light curves. During a major portion of the huge radio flares associated with superluminal blob emission a series of soft dips are present (Naik et al. 2001). Mirabel \& Rodriguez (1999) have pointed out that in each epoch of this type of outflow, the mass condensation is around $10^{23} \mathrm{~g}$. In order to achieve this, we require that matter is accumulated from disk evacuation at each "dip" and within each epoch, successive mini-blobs move faster than the earlier blob in order to "catch up". This may indicate some other runaway process with a longer time scale of tens of days. Naik et al. (2001) have observed such X-ray dips at a rate of once in a few hundred seconds during the peak or the radio flare. If there are $\sim 1000$ evacuation events during an episode of superluminal blob ejection (in a few days), then total mass condensation would be $10^{23} \mathrm{~g}$. Hence in order to explain the observation of Mirabel \& Rodriguez (1999), one must require that in each epoch, matter is accumulated from at least a thousand evacuation events. Future observation would tell if such is the case.

It is to be noted that the mass of the condensation as estimated by Mirabel \& Rodriguez (1999) is based on the presence of one electron per proton, i.e., no pair production is assumed. With a pair density, say, ten times larger, the estimated mass would be ten times less. However, at the same time, estimated mass of the disk would also be reduced by the same factor. Hence, the number of ejection events is not affected.

Acknowledgements. SKC and AN acknowledge receiving grants from DST project entitled Analytical and Numerical 
Studies of Astrophysical Flows Around Compact Objects. The authors thank the referee for helpful comments.

\section{References}

Balbus, S. A., \& Hawley, J. F., 1991, ApJ, 376, 214

Belloni, T., Mendez, M., King, A. R., van der Klis, M., \& van Paradijs, J. 1997, ApJ, 479, L145

Belloni, T., Klein-Wolt, M., Mendez, M., van der Klis, M., \& van Paradijs, J. 2000, A\&A, 355, 271

Castro-Tirado, A. J., Brandt, S., \& Lund, N. 1992, IAU Circ., 5590

Chakrabarti, S. K. 1990, Theory of Transonic Astrophysical Flows (World Scientific: Singapore)

Chakrabarti, S. K. 1994, Proc. of 17th Texas Symp., ed. H. Böhringer, E. Morfill, \& J. Trümper (New York Academy of Sciences, New York), 546

Chakrabarti, S. K. 1996a, AJ, 464, 664

Chakrabarti, S. K. 1996b, Phys. Rep., 266, 318

Chakrabarti, S. K. 2000 Class. Quant. Grav., 17, 2427

Chakrabarti, S. K., \& D'Silva, S. 1994, ApJ, 424, 138 (CD94)

Chakrabarti, S. K., Titarchuk, L. G., Kazanas, D., \& Ebisawa, K. 1996 A\&AS, 120, 163

Chakrabarti, S. K., Manickam, S., Nandi, A., \& Rao, A. R. 2001 in Proc. of the IXth Marcel Grossman meeting, ed. R. Ruffini (World Scientific Press: Singapore), in press

Chakrabarti, S. K., \& Manickam, S. 2000, ApJ, 531, L41 (CM00)

Chakrabarti, S. K., \& Titarchuk, L. G. 1995, ApJ, 455, 623

Dhawan, V., Mirabel, I. F., \& Rodriguez, L. F. 2000, ApJ, 543, 373

D’Silva, S., \& Chakrabarti, S. K. 1994, ApJ, 424, 149

Fender, R. P., Pooley, G., Brocksopp, C., \& Newell, S. J. 1997, MNRAS, 290, L65

Fender, R. P., Garrington, S. T., McKay, D. J., et al. 1999, MNRAS, 304, 865

Feroci, M., Matt, G., Pooley, G., et al. 1999, A\&A, 351, 985
Goldstein, S. 1938, in Modern Developments in Fluid Mechanics (Oxford: Clarendon Press)

Foglizzo, T., \& Tagger, M. 1995, A\&A, 301, 293

Hawley, J. F., Gammie, C. F., \& Balbus, S. A. 1995, ApJ, 440, 742

Mirabel, I. F., \& Rodriguez, L. F. 1994, Nature, 371, 46

Mirabel, I. F., \& Rodriguez, L. F. 1999, ARA\&A, 37, 409

Mirabel, I. F., Dhaman, V., Chaty, S., et al. 1998, A\&A, 330, L9

Molteni, D., Sponholz, H., \& Chakrabarti, S. K. 1996, ApJ, 457,805

Morgan, E. H., Remillard, R. A., \& Greiner, J. 1997, ApJ, 482, 993

Muno, M. P., Morgan, E. H., \& Remillard, R. A. 1999, ApJ, 527,321

Naik, S., Agrawal, P. C., Rao, A. R., Paul, B., et al. 2001, ApJ, 546, 1075

Naik, S., \& Rao, A. R. 2000, A\&A, 362, 691

Paczynśki, B., \& Wiita, P. J. 1980, A\&A, 88, 23

Parker, E. N. 1979, Cosmical Magnetic Fields (Clarendon press, Oxford)

Pooley, G. G., \& Fender, R. P. 1997, MNRAS, 292, 925

Rao, A. R., Naik, S., Vadawale, S. V., \& Chakrabarti, S. K. 2000, A\&A, 360, L25

Ryu, D., Chakrabarti, S. K., \& Molteni, D. 1997, ApJ, 474, 378

Shakura, N. I., \& Sunyaev, R. A. 1973, A\&A, 24, 337

Shimura, T., \& Takahara, F. 1995, ApJ, 445, 780

Smith, D. M., Heindl, W. A., \& Swank, J. H. 2001a, ApJ, submitted

Smith, D. M., Heindl, W. A., Markwardt, C. B., \& Swank, J. H. 2001b, ApJ, 554, 41

Vadawale, S. V., Rao, A. R., Nandi, A., \& Chakrabarti, S. K. 2001, A\&A, 370, L17

Yadav, J. S., Rao, A. R., Agrawal, P. C., et al. 1999, ApJ, 517, 935 